See Article page 463.

\section{Commentary: New players in epidermal growth factor receptor tyrosine kinase inhibitor resistance}

\author{
Elise Sienicki, MD, ${ }^{\mathrm{a}}$ and Mark Onaitis, $\mathrm{MD}^{\mathrm{b}}$
}

Targeted inhibition of the epidermal growth factor receptor (EGFR) tyrosine kinase in lung adenocarcinoma is limited by acquired resistance. Better understanding of the development of this resistance is a significant unmet need in lung adenocarcinoma treatment. The group at Medical University of South Carolina in the present study ${ }^{1}$ has identified a mechanism for resistance in EGFR mutant lung adenocarcinoma that relies on the NRP2B-GSK3B-PTEN-AKT axis. This group has previously demonstrated that NPR2B is absent from normal lung tissue and upregulated in lung cancer and that its presence correlates with poor clinical outcomes. $^{2}$ The present study builds on the previous work. First, shRNA inhibition of NRP2B led to 100-fold decrease in persister cells after gefitinib treatment. In addition, NRP2B knockdown can sensitize some EGFR wild-type cells to gefitinib. In HEK293 cells, expression of NRP2B leads to greater phosphorylation of phosphatase and tensin homolog (PTEN) and its subsequent posttranslational degradation. NRP2B binds glycogen synthase kinase 3 beta $(\mathrm{GSK} 3 \beta)$ preferentially with subsequent GSK3 $\beta$-dependent phosphorylation of PTEN. Migration and development of persister cells are dependent on GSK $3 \beta$ signaling. Importantly for therapeutic development, lack of NRP2B is well tolerated by cells. These are important findings that will help the EGFR resistance field to move forward.

\footnotetext{
From the ${ }^{\mathrm{a}}$ Department of Surgery, Naval Medical Center; and ${ }^{\mathrm{b}}$ Division of Cardiothoracic Surgery, University of California San Diego, San Diego, Calif.

Disclosures: The authors reported no conflicts of interest.

The Journal policy requires editors and reviewers to disclose conflicts of interest and to decline handling or reviewing manuscripts for which they may have a conflict of interest. The editors and reviewers of this article have no conflicts of interest.

Received for publication April 21, 2020; accepted for publication April 23, 2020; available ahead of print May 4, 2020.

Address for reprints: Mark Onaitis, MD, Division of Cardiothoracic Surgery, University of California San Diego, 9300 Campus Point Dr, Mail Code 7892, La Jolla, CA 92037 (E-mail: monaitis@ucsd.edu).

J Thorac Cardiovasc Surg 2021;162:474-5 $0022-5223 / \$ 36.00$

Copyright $₫ 2020$ Published by Elsevier Inc. on behalf of The American Association for Thoracic Surgery

https://doi.org/10.1016/j.jtcvs.2020.04.096
}

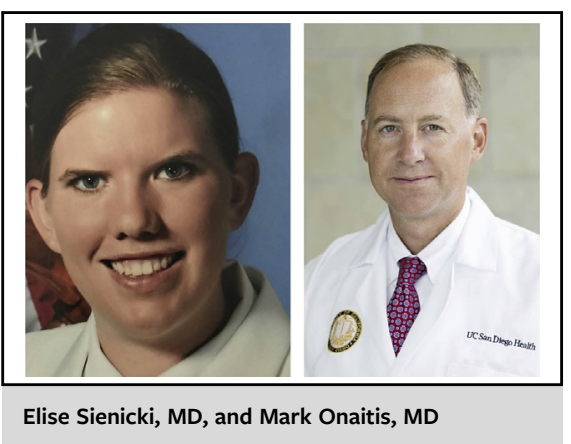

CENTRAL MESSAGE

Resistance to EGFR tyrosine kinase inhibitors is complex and will require multidisciplinary teams to develop effective treatment regimens.

The field of tyrosine kinase inhibitor (TKI) resistance is very complex. Although EGFR inhibition has represented a great advance in lung cancer therapy, acquired resistance has limited its effectiveness in improving long-term survival rates. Many mechanisms of resistance have been described. Most commonly, the development of T790M mutation renders the EGFR protein resistant to first- and second-generation TKIs (gefitinib, erlotinib, icotinib, afatinib). Recently, the third-generation TKI osimertinib has been demonstrated to be effective in EGFR mutant lung adenocarcinoma even in the first-line setting. ${ }^{3}$ However, other resistance mechanisms representing alternative downstream signaling pathways are common even after osimertinib treatment and must be addressed. These include MET activation, phosphoinositide 3-kinase (PI3K) pathway activation, and KRAS activation, among others. ${ }^{4}$

One of the strengths of the current work is that some of these clinical issues are addressed. Osimertinib is used to address resistance to third-generation inhibitors. The mechanism proposed in the current article acts through PTEN/ AKT, which are downstream effectors of PI3K. These careful, well-controlled signal transduction experiments are being performed by a multidisciplinary team of scientists and surgeon-scientists.

As the authors acknowledge, work such as this raises many new questions that must be addressed to achieve clinical relevance. These mechanisms must be validated using in vivo models. In terms of planning eventual treatment, the question of whether NR2B inhibition or downstream PI3K signaling should be targeted must be answered. For surgeons, whether EGFR inhibition or EGFR inhibition in 
tandem with resistance inhibitors is appropriate in an adjuvant setting or in an induction setting will be important issues to address. The National Cancer Institute's ALCHEMIST (Adjuvant Lung Cancer Enrichment Marker Identification and Sequencing Trial) trial only uses the first-generation TKI erlotinib in EGFR-mutant patients. Finally, at present, immunotherapy approaches are not effective in patients with EGFR mutations. Whether programmed cell death protein 1/programmed death-ligand 1 expression and tumor mutational burden are increased when NR2B is upregulated will be an interesting question.

In summary, this is a very interesting study that presents a novel resistance mechanism of EGFR TKI resistance. Elegant signal transduction experiments are described that may eventually lead to therapeutic advances. Surgeons must be involved in and aware of this type of research so that we can develop new therapies for these patients and communicate effectively with oncologists and patients.

\section{References}

1. Dimou A, Nasarre C, Peterson YK, Pagano R, Gooz M, Nasarre P, et al. Neuropilin-2b facilitates resistance to tyrosine kinase inhibitors in non-small cell lung cancer. J Thorac Cardiovasc Surg. 2021;162:474-5.

2. Gemmill RM, Nasarre P, Nair-Menon J, Cappuzzo F, Landi L, D'Incecco A, et al The neuropilin 2 isoform NRP2B uniquely supports TGFbeta-mediated progression in lung cancer. Sci Signal. 2017; 10:eaag0528.

3. Ramalingam SS, Yang JC, Lee CK, Kurata T, Kim DW, John T, et al. Osimertinib as first-line treatment of EGFR mutation-positive advanced non-small-cell lung cancer. J Clin Oncol. 2018;36:841-9.

4. Chabon JJ, Simmons AD, Lovejoy AF, Esfahani MS, Newman AM, Haringsma HJ, et al. Circulating tumor DNA profiling reveals heterogeneity of EGFR inhibitor resistance mechanisms in lung cancer patients. Nat Commun. 2016;7:1-14.
See Article page 463.

\section{Commentary: Keeping the target painted for precision cancer therapy}

\author{
David S. Schrump, MD, MBA, and \\ Chuong D. Hoang, MD
}

Observations that activating mutations in the epidermal growth factor receptor (EGFR) gene confer sensitivity to EGFR-tyrosine kinase inhibitors (EGFR-TKIs) such as gefitinib and erlotinib ushered in the modern era of targeted therapy for non-small cell lung cancer (NSCLC). ${ }^{1,2}$ EGFR mutations occur in approximately $20 \%$ of NSCLC, typically in female patients, nonsmokers, and patients

\footnotetext{
From the Thoracic Epigenetics Section, Thoracic Surgery Branch, Center for Cancer Research, National Cancer Institute, Bethesda, Md.

Disclosures: The authors reported no conflicts of interest.

The Journal policy requires editors and reviewers to disclose conflicts of interest and to decline handling or reviewing manuscripts for which they may have a conflict of interest. The editors and reviewers of this article have no conflicts of interest.

Received for publication April 18, 2020; accepted for publication April 20, 2020; available ahead of print May 4, 2020.

Address for reprints: David S. Schrump, MD, MBA, Thoracic Epigenetics Section, Thoracic Surgery Branch, Center for Cancer Research/NCI, 10 Center Dr, 43942, Bethesda, MD 20892 (E-mail: David_Schrump@nih.gov).

J Thorac Cardiovasc Surg 2021;162:475-6 0022-5223/\$0.00

Published by Elsevier Inc. on behalf of The American Association for Thoracic Surgery

https://doi.org/10.1016/j.jtcvs.2020.04.095
}

Check for updates

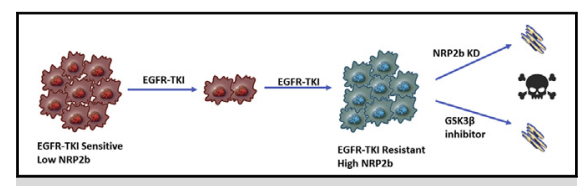

Resistance to EGFR-TKI coincides with and depends on up-regulation of NRP2b.

\begin{abstract}
CENTRAL MESSAGE
Acquired resistance to EGFR-TKI

in NSCLC is associated with upregulation of NRP2b. Targeting

NRP2b may be a novel strategy

to restore and maintain sensi-

tivity to these agents for lung

cancer therapy.
\end{abstract}

with adenocarcinomas. ${ }^{3}$ Despite impressive initial responses, virtually all patients develop resistance to EGFRTKI due to secondary mutations in EGFR, mutations that activate survival signaling pathways such as $M E T$, evolution of a small cell lung cancer phenotype, or epithelial-tomesenchymal transition (EMT). ${ }^{4}$ As such, delineation of mechanisms conferring resistance to EGFR-TKI is a major focus of investigative efforts in thoracic oncology.

In this issue of the Journal, Dimou and colleagues ${ }^{5}$ examined the role of Neuropilin-2b (NRP2b) in acquired resistance to EGFR-TKI in NSCLC. Previous studies by this 